\title{
Consensus Optimization for Distributed Registration
}

\author{
Rajat Sanyal ${ }^{\dagger}$ and Kunal N. Chaudhury ${ }^{\star}$
}

${ }^{\dagger}$ IGH-S\&O, KPMG Advisory Services Private Limited, Gurugram, India

${ }^{\star}$ Department of Electrical Engineering, Indian Institute of Science, Bengaluru, India

\section{GlobalSIP-2018}




\section{Registration Problem}

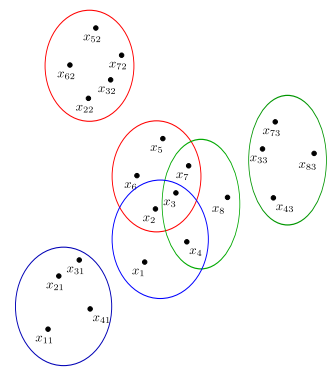

Figure: Registration problem for three point clouds.

- Given: Local coordinates $\mathbf{x}_{k, i}$, point correspondence.

- Unknowns: Global coordinates $\mathbf{z}_{1}, \ldots, \mathbf{z}_{N}$, rigid transformations $\left(\mathbf{O}_{1}, \mathbf{t}_{1}\right), \ldots,\left(\mathbf{O}_{M}, \mathbf{t}_{M}\right)$ where $\mathbf{O}_{i} \in \mathbb{O}(d), \mathbf{t} \in \mathbb{R}^{d}$, and $\mathbb{O}(d)=\left\{\mathbf{O} \in \mathbb{R}^{d \times d}: \mathbf{O}^{\top} \mathbf{O}=\mathbf{I}_{d}\right\}$.

- Noiseless scenario: $\mathbf{z}_{k}=\mathbf{O}_{i} \mathbf{x}_{k, i}+\mathbf{t}_{i}$. 


\section{Applications}

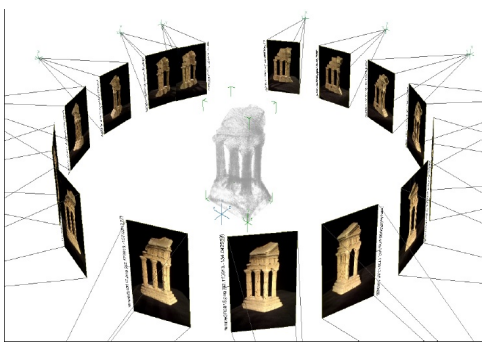

(a) Multiview registration.

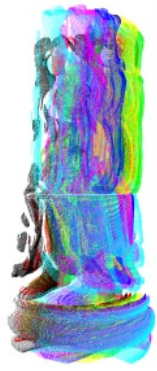

(b) 3D scan.

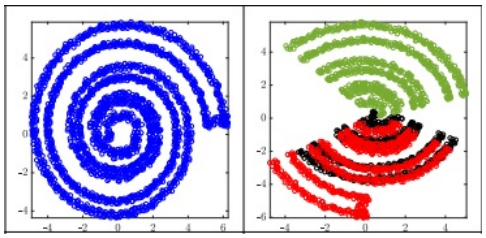

(c) Sensor network localization. 


\section{Sensor Network Localization (SNL)}

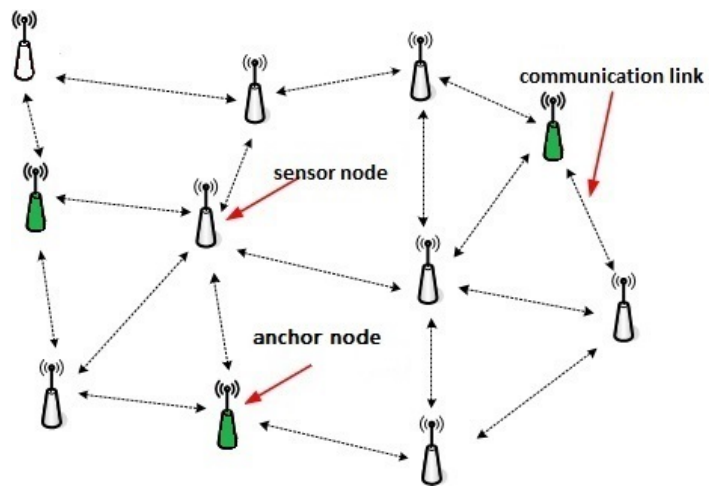

- Available information: Inter-sensor distances.

- Aim: Estimate the original location of the sensors, or up to some rigid transformation (rotation, reflection, translation) of the original locations. 


\section{Divide-and-Conquer Based SNL Algorithm}
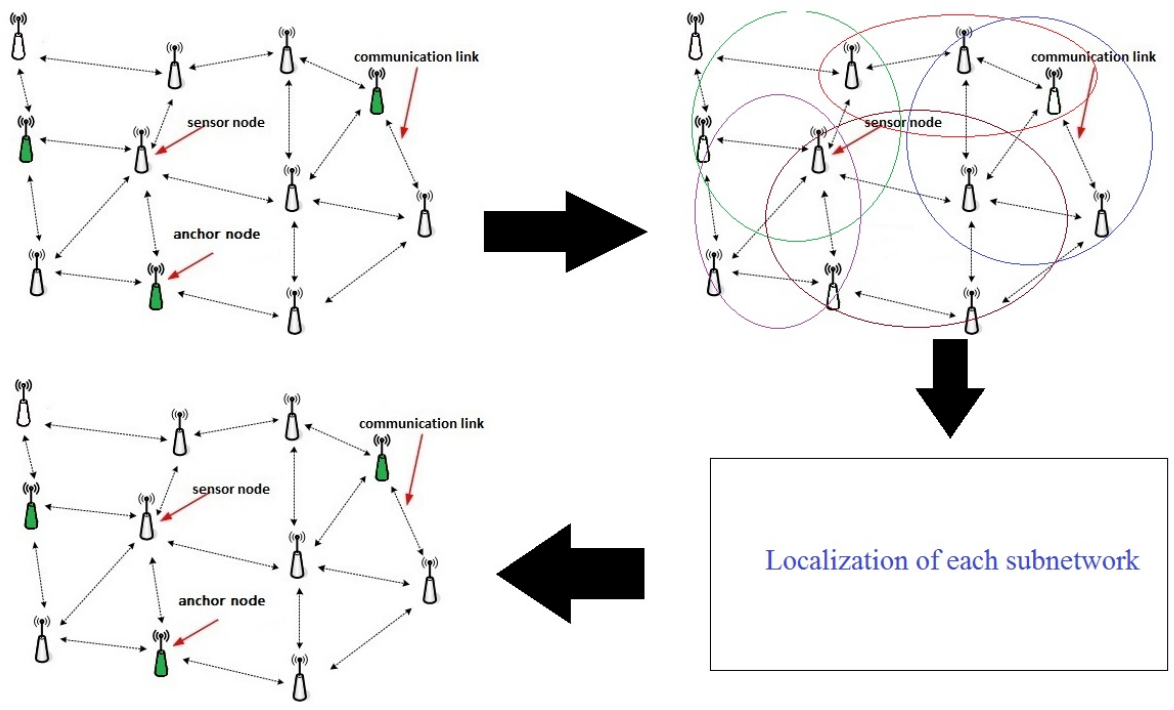


\section{Least Square Formulation}

$$
\min _{\mathbf{z}_{k}, \mathbf{t}_{i} \in \mathbb{R}^{n}, \mathbf{O}_{i} \in \mathbb{O}(d)} \sum_{i=1}^{M} \sum_{k \in \mathcal{P}_{i}}\left\|\mathbf{z}_{k}-\left(\mathbf{O}_{i} \mathbf{x}_{k, i}+\mathbf{t}_{i}\right)\right\|^{2} .
$$

- Non-convex problem.

- Convex relaxation proposed by Chaudhury et al., SIOPT $2015^{1}$ :

- Fix $\mathbf{O}_{i}{ }^{\prime}$ s, jointly optimizes over $\mathbf{x}_{k}$ and $\mathbf{t}_{i}$.

- Leads to the following problem

$$
\begin{array}{ll}
\min _{\mathbf{G} \in \mathbb{S}_{+}^{n}} & \operatorname{Tr}(\mathbf{C G}) \\
\text { s.t. } & {[\mathbf{G}]_{i i}=\mathbf{I}_{d}, \forall i \in[1: M], \operatorname{rank}(\mathbf{G})=d .}
\end{array}
$$

- Drop the rank and solve the semidefinite programming.

${ }^{1}$ K. N. Chaudhury, Y. Khoo, and A. Singer, "Global registration of multiple point clouds using semidefinite programming," SIAM Journal on Optimization, vol. 25, no. 1, pp. 468-501, 2015. 


\section{What is the issue then?}

- Computing C.

$$
\mathbf{C}=\mathbf{D}-\mathbf{B L}^{\dagger} \mathbf{B}^{\top}
$$

$\mathbf{L}$ is a symmetric matrix of size $(N+M)$.

- Large number of point clouds.

- Rank of $\mathbf{G}^{\star}$ may not be $d$. 


\section{Repose the Registration Problem}

- The least-square formulation of the registration problem:

$$
\min _{\mathbf{z}_{k}, \mathbf{t}_{i} \in \mathbb{R}^{n}, \mathbf{O}_{i} \in \mathbb{O}(d)} \sum_{i=1}^{M} \sum_{k \in \mathcal{P}_{i}}\left\|\mathbf{z}_{k}-\left(\mathbf{O}_{i} \mathbf{x}_{k, i}+\mathbf{t}_{i}\right)\right\|^{2}
$$

- Reformulate the registration problem:

$$
\begin{array}{cl}
\min _{\mathbf{y}_{k, i}, \mathbf{z}_{k}, \mathbf{t}_{i} \in \mathbb{R}^{n}, \mathbf{O}_{i} \in \mathbb{O}(d)} & \sum_{i=1}^{M} \sum_{k \in \mathcal{P}_{i}}\left\|\mathbf{y}_{k, i}-\left(\mathbf{O}_{i} \mathbf{x}_{k, i}+\mathbf{t}_{i}\right)\right\|^{2} \\
\text { s.t. } & \mathbf{y}_{k, i}=\mathbf{z}_{k}, \forall k \in \mathcal{P}_{i}, \quad i \in[1: M] .
\end{array}
$$




\section{Membership Graph}
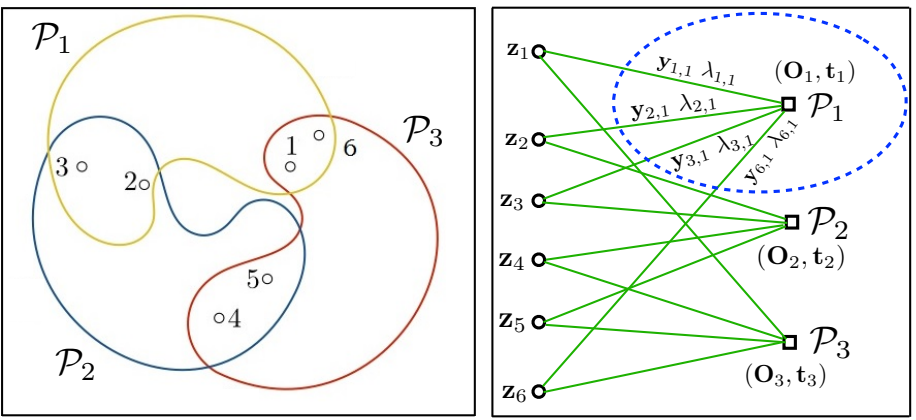

An example of a point set configuration, and the correspondence graph.

$$
\begin{array}{cl}
\min _{\mathbf{y}_{k, i}, \mathbf{z}_{k}, \mathbf{t}_{i} \in \mathbb{R}^{n}, \mathbf{O}_{i} \in \mathbb{O}(d)} & \sum_{i=1}^{M} \sum_{k \in \mathcal{P}_{i}}\left\|\mathbf{y}_{k, i}-\left(\mathbf{O}_{i} \mathbf{x}_{k, i}+\mathbf{t}_{i}\right)\right\|^{2} \\
\text { s.t. } & \mathbf{y}_{k, i}=\mathbf{z}_{k}, \quad \forall k \in \mathcal{P}_{i}, \quad i \in[1: M] .
\end{array}
$$




\section{Augmented Lagrangian and the ADMM Solver}

- Problem:

$$
\begin{array}{cc}
\min _{\mathbf{y}_{k, i}, \mathbf{z}_{k}, \mathbf{t}_{i} \in \mathbb{R}^{n}, \mathbf{O}_{i} \in \mathbb{O}(d)} & \sum_{i=1}^{M} \sum_{k \in \mathcal{P}_{i}}\left\|\mathbf{y}_{k, i}-\left(\mathbf{O}_{i} \mathbf{x}_{k, i}+\mathbf{t}_{i}\right)\right\|^{2} \\
\text { s.t. } & \mathbf{y}_{k, i}=\mathbf{z}_{k}, \forall k \in \mathcal{P}_{i}, \quad i \in[1: M] .
\end{array}
$$

- Augmented Lagrangian:

$$
\mathcal{L}_{\rho}=\sum_{k \sim i}\left(\left\|\mathbf{y}_{k, i}-\left(\mathbf{O}_{i} \mathbf{x}_{k, i}+\mathbf{t}_{i}\right)\right\|^{2}+\lambda_{k, i}^{\top}\left(\mathbf{y}_{k, i}-\mathbf{z}_{k}\right)+\frac{\rho}{2}\left\|\mathbf{y}_{k, i}-\mathbf{z}_{k}\right\|^{2}\right) .
$$

- Alternating direction methods for multipliers (ADMM) solver:

$$
\begin{aligned}
& \left(\mathbf{Y}^{(t)}, \mathbf{O}^{(t)}, \mathbf{T}^{(t)}\right)=\underset{\mathbf{Y}, \mathbf{O}, \mathbf{T}}{\operatorname{argmin}} \mathcal{L}_{\rho}\left(\mathbf{Y}, \mathbf{O}, \mathbf{T}, \mathbf{Z}^{(t-1)}, \mathbf{\Lambda}^{(t-1)}\right), \\
& \mathbf{Z}^{(t)}=\underset{\mathbf{Z}}{\operatorname{argmin}} \mathcal{L}_{\rho}\left(\mathbf{Y}^{(t)}, \mathbf{O}^{(t)}, \mathbf{T}^{(t)}, \mathbf{Z}, \mathbf{\Lambda}^{(t-1)}\right), \\
& \boldsymbol{\lambda}_{k, i}^{(t)}=\boldsymbol{\lambda}_{k, i}^{(t-1)}+\rho\left(\mathbf{y}_{k, i}^{(t)}-\mathbf{z}_{k}^{(t)}\right), \quad(k \sim i) .
\end{aligned}
$$




\section{Update $\mathbf{Y}, \mathbf{O}, \mathbf{T}$}

$\min _{\mathbf{y}_{k, i}, \mathbf{t}_{i}, \mathbf{O}_{i}} \sum_{i=1}^{M} \sum_{k \in \mathcal{P}_{i}}\left(\left\|\mathbf{y}_{k, i}-\left(\mathbf{O}_{i} \mathbf{x}_{k, i}+\mathbf{t}_{i}\right)\right\|^{2}+\frac{\rho}{2}\left\|\mathbf{y}_{k, i}-\left(\mathbf{z}_{k}-\lambda_{k, i} / \rho\right)\right\|^{2}\right)$

- For each point cloud:

$$
\min _{\mathbf{y}_{k, i}, \mathbf{t}_{i}, \mathbf{O}_{i}} \sum_{k \in \mathcal{P}_{i}}\left(\left\|\mathbf{y}_{k, i}-\left(\mathbf{O}_{i} \mathbf{x}_{k, i}+\mathbf{t}_{i}\right)\right\|^{2}+\frac{\rho}{2}\left\|\mathbf{y}_{k, i}-\left(\mathbf{z}_{k}-\lambda_{k, i} / \rho\right)\right\|^{2}\right)
$$

- First, minimizes over $\boldsymbol{y}_{k, i}$ and $\mathbf{t}_{i}$.

- Finally, solve the following:

$$
\max _{\mathbf{O}_{i} \in \mathbb{O}(d)} \operatorname{Tr}\left(\mathbf{C}_{i} \mathbf{O}_{i}\right) .
$$




\section{Update $\mathbf{Z}$}

$$
\min _{\mathbf{z}_{k}} \sum_{k \sim i}\left\|\mathbf{z}_{k}-\left(\mathbf{y}_{k, i}^{(t)}+\rho^{-1} \boldsymbol{\lambda}_{k, i}^{(t-1)}\right)\right\|^{2}
$$
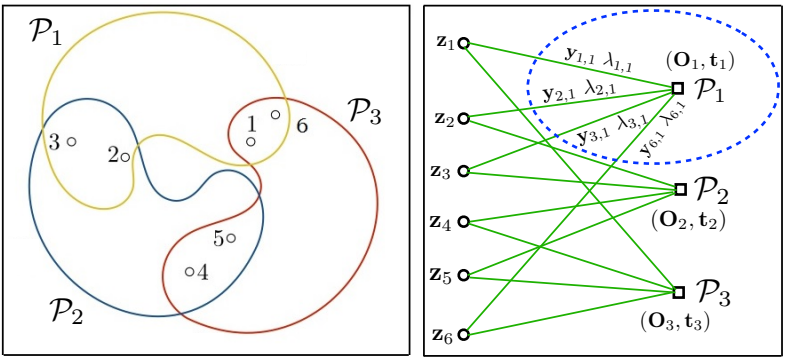

$$
\mathbf{z}_{k}^{(t)}=\frac{1}{\left|\mathcal{N}_{k}\right|} \sum_{i \in \mathcal{N}_{k}}\left(\boldsymbol{y}_{k, i}^{(t)}+\rho^{-1} \boldsymbol{\lambda}_{k, i}^{(t-1)}\right)
$$




\section{Summary}

- Computation is distributed over each point-cloud.

- Main computation per processor is an SVD: $\mathcal{O}\left(d^{3}\right)$.

- Solve the non-convex problem directly. 


\section{Performance Metric}

- Performance metric: Average Normalized Error² (ANE).

$$
\operatorname{ANE}=\left\{\frac{\sum_{i=1}^{N}\left\|\hat{\mathbf{x}}_{i}-\overline{\mathbf{x}}_{i}\right\|^{2}}{\sum_{i=1}^{N}\left\|\overline{\mathbf{x}}_{i}-\overline{\mathbf{x}}_{c}\right\|^{2}}\right\}^{1 / 2}
$$

where,

$\hat{\mathbf{x}}_{i}$ : estimated sensor position after the alignment,

$\mathbf{x}_{i}$ : actual position of the sensor,

$\overline{\mathbf{x}}_{c}$ : the centroid of the original sensor positions

$$
\overline{\mathbf{x}}_{c}=\frac{1}{N} \sum_{i=1}^{N} \overline{\mathbf{x}}_{i}
$$

${ }^{2}$ M. Cucuringu, Y. Lipman, and A. Singer, "Sensor network localization by eigenvector synchronization over the Euclidean group," ACM Trans. on Sensor Networks, vol. 8, no. 3, pp. 19-42, 2012. 


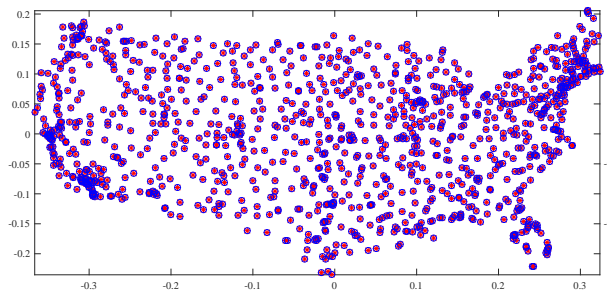

(d) $\eta=0, \mathrm{ANE}=9.5 \mathrm{e}-13$.

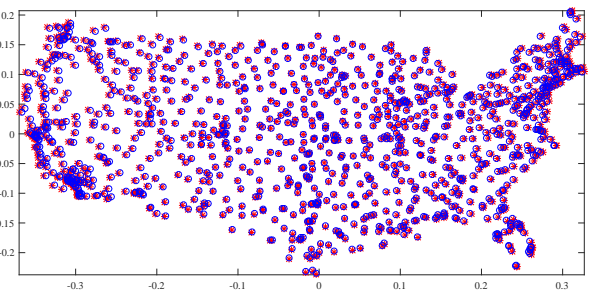

(e) $\eta=0.006, \mathrm{ANE}=9.1 \mathrm{e}-3$.

Localization of the US cities dataset consisting of 1101 points. The sensing radius used for both (a) and (b) is $r=0.06$, which is about $9 \%$ of the diameter of the dataset (0.704). The original and estimated locations are marked using blue circles and red stars. 


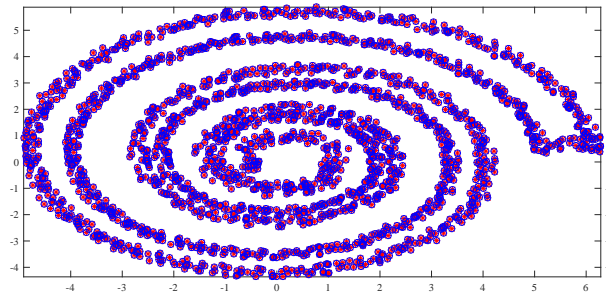

(f) $\eta=0, \mathrm{ANE}=4.6 \mathrm{e}-11$.

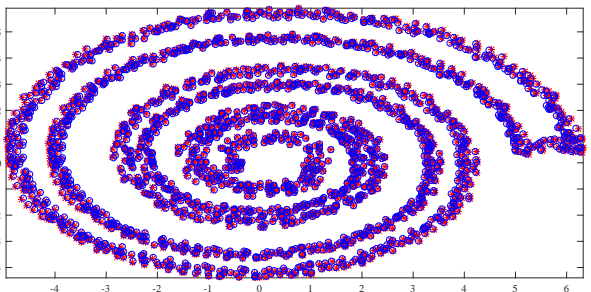

(g) $\eta=0.01, \mathrm{ANE}=7.6 \mathrm{e}-3$.

Localization of the spiral dataset consisting of 2259. The sensing radius used for both (a) and (b) is $r=1$, which is about $9 \%$ of the diameter of the dataset (11.2). The original and estimated locations are marked using blue circles and red stars. 


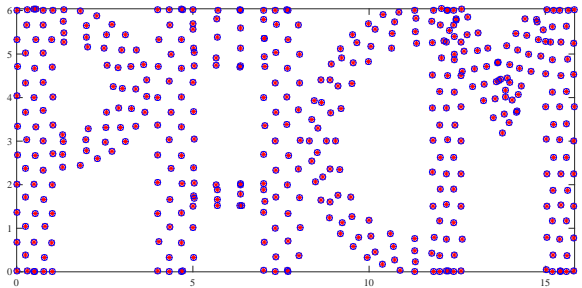

(h) Proposed (ANE $=4.4 \mathrm{e}-12$ ).

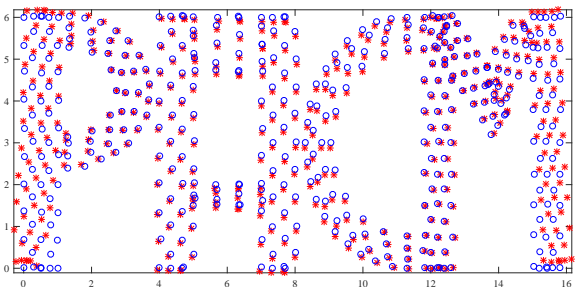

(i) $\operatorname{SNLSDP}$ (ANE $=2.9 \mathrm{e}-2$ )

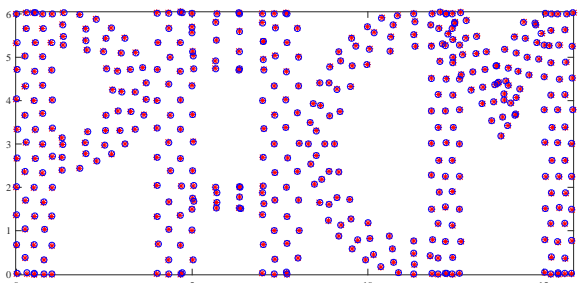

(j) Proposed (ANE $=2.6 \mathrm{e}-3$ ).

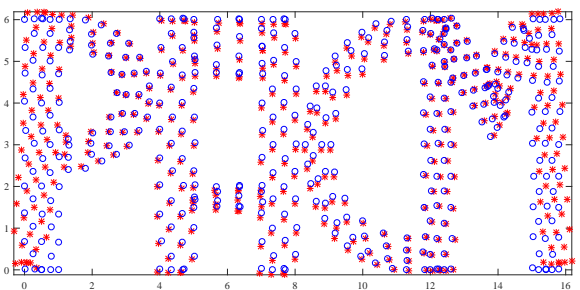

(k) SNLSDP (ANE $=3 \mathrm{e}-2$ )

Localization of PACM dataset consisting of 495 points. The top and bottom rows correspond to $\eta=0$ and $\eta=0.03$. The original and estimated locations are marked using blue circles and red stars. 


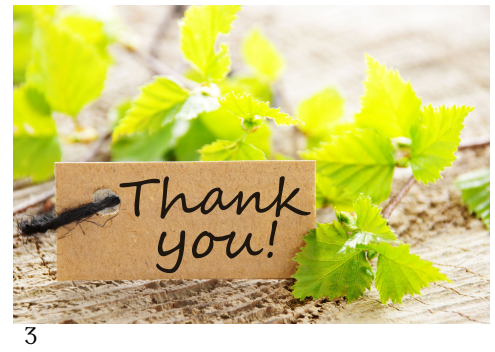

\section{Questions?}

